\title{
Stability of a functional equation connected with Reynolds operator
}

Jaeyoung Chung*

\section{*Correspondence:}

jychung@kunsan.ac.kr

Department of Mathematics,

Kunsan National University, Kunsan,

573-701, Korea

\begin{abstract}
Let $G$ be a commutative semigroup, $\mathbb{K}=\mathbb{R}$ or $\mathbb{C}$ and $F: G \rightarrow \mathbb{K}^{n}$. Generalizing the stability of the functional equation $F(x \circ g(y))-F(x) F(y)=0$ with bounded difference (Najdecki in J. Inequal. Appl. 2007:79816, 2007), we prove the stability of the above functional equation with unbounded differences. We also give a more precise description for bounded components of $F=\left(f_{1}, f_{2}, \ldots, f_{n}\right)$.
\end{abstract}

MSC: 39B82

Keywords: bounded solution; exponential function; involution; Reynolds operator; stability

\section{Main results}

Throughout this paper, $\langle G, \circ\rangle$ is a commutative semigroup with an identity $e, \mathbb{R}$ the set of real numbers, $\mathbb{C}$ the set of complex numbers, $\mathbb{K}=\mathbb{R}$ or $\mathbb{C}, \epsilon \geq 0$, and $g: G \rightarrow G$ and $\phi: G \rightarrow[0, \infty)$ are given functions. For $\left(a_{1}, a_{2}, \ldots, a_{n}\right),\left(b_{1}, b_{2}, \ldots, b_{n}\right) \in \mathbb{K}^{n}$, we define $\left(a_{1}, a_{2}, \ldots, a_{n}\right)\left(b_{1}, b_{2}, \ldots, b_{n}\right)=\left(a_{1} b_{1}, a_{2} b_{2}, \ldots, a_{n} b_{n}\right)$. A function $\sigma: G \rightarrow G$ is said to be $a n$ involution if $\sigma(x \circ y)=\sigma(x) \circ \sigma(y)$ and $\sigma(\sigma(x))=x$ for all $x, y \in G$. A function $m: G \rightarrow \mathbb{K}^{n}$ is called an exponential function provided that $m(x \circ y)=m(x) m(y)$ for all $x, y \in G$.

Generalizing the result of Ger and Šemrl [1], Najdecki [2] proved the stability of the functional equation

$$
F(x \circ g(y))-F(x) F(y)=0
$$

in the class of functions $F: G \rightarrow \mathbb{K}^{n}$. The particular cases of (1.1) are the exponential equation $f(x y)=f(x) f(y)$ (see Aczél and Dhombres [3] and Baker [4]) and the equation

$$
f(x f(y))=f(x) f(y)
$$

for all $x, y \in \mathbb{K} \backslash\{0\}$, where $f: \mathbb{K} \backslash\{0\} \rightarrow \mathbb{K} \backslash\{0\}$ (see Brzdęk [5], Brzdęk, Najdecki and $\mathrm{Xu}$ [6] and Chudziak and Tabor [7] for related equations). As mentioned in [2, 5], (1.2) arises in averaging theory applied to the turbulent fluid motion and is connected with the Reynolds operator (see Marias [8]), the averaging operator and the multiplicatively symmetric operator (see [3]). Moreover, the equation (1.2) is connected with a description of some associative operations, i.e., the binary operation $\circ:(\mathbb{K} \backslash\{0\}) \times(\mathbb{K} \backslash\{0\}) \rightarrow \mathbb{K} \backslash\{0\}$ defined by $x \circ f(y)=x f(y)$ is associative if and only if $f$ satisfies (1.2) (see [5] for more 
details). We also refer the reader to Belluot, Brzdęk and Ciepliński [9] and Brzdęk and Ciepliński [10] for some recent developments on the issues of stability and superstability for functional equations.

The main result of Najdecki [2] is the following.

Theorem 1.1 Let $F: G \rightarrow \mathbb{K}^{n}, F=\left(f_{1}, f_{2}, \ldots, f_{n}\right)$ satisfy

$$
\|F(x \circ g(y))-F(x) F(y)\| \leq \epsilon
$$

for all $x, y \in G$ with any norm $\|\cdot\|$ in $\mathbb{K}^{n}$. Then there exist ideals $I, J \subset \mathbb{K}^{n}$ such that $\mathbb{K}^{n}=$ $I \oplus J, P F$ is bounded and $Q F$ satisfies (1.1), where $P: \mathbb{K}^{n} \rightarrow I, Q: \mathbb{K}^{n} \rightarrow J$ are the natural projections.

In this paper, generalizing the above result we consider the functional inequalities

$$
\begin{aligned}
& \|F(x \circ g(y))-F(x) F(y)\| \leq \phi(y), \\
& \|F(x \circ g(y))-F(x) F(y)\| \leq \phi(x)
\end{aligned}
$$

for all $x, y \in G$ with any norm $\|\cdot\|$ in $\mathbb{K}^{n}$ (see [6] for related results).

Throughout this paper we denote

$$
\begin{aligned}
& L=\left\{j: f_{j} \text { is bounded, } j=1,2, \ldots, n\right\}, \\
& K=\left\{j: f_{j} \text { is unbounded, } j=1,2, \ldots, n\right\},
\end{aligned}
$$

where $F=\left(f_{1}, f_{2}, \ldots, f_{n}\right)$.

Theorem 1.2 Let $F: G \rightarrow \mathbb{K}^{n}, F=\left(f_{1}, f_{2}, \ldots, f_{n}\right)$ satisfy $(1.4)$ for all $x, y \in G$ with any norm $\|\cdot\|$ in $\mathbb{K}^{n}$. Assume that one of the following two conditions is fulfilled.

(i) $g$ is an involution,

(ii) for each $j \in K$, there exists a sequence $x_{n}, n=1,2,3, \ldots$ (possibly depending on $j$ ) such that

$$
\frac{\left|f_{j}\left(x_{n}\right)\right|}{1+\phi\left(x_{n}\right)} \rightarrow \infty \quad \text { as } n \rightarrow \infty .
$$

Then there exist ideals $I, J \subset \mathbb{K}^{n}$ such that $\mathbb{K}^{n}=I \oplus J$, PF is bounded and $Q F$ satisfies (1.1), where $P: \mathbb{K}^{n} \rightarrow I, Q: \mathbb{K}^{n} \rightarrow J$ are the natural projections. Moreover, $Q\left(F \circ g^{-1}\right)$ is exponential provided $g$ is bijective.

Remark The case (ii) of Theorem 1.2 includes Theorem 1.1.

Theorem 1.3 Let $F: G \rightarrow \mathbb{K}^{n}, F=\left(f_{1}, f_{2}, \ldots, f_{n}\right)$ satisfy $(1.5)$ for all $x, y \in G$ with any norm $\|\cdot\|$ in $\mathbb{K}^{n}$. Assume that $g$ is an involution. Then there exist ideals $I, J \subset \mathbb{K}^{n}$ such that $\mathbb{K}^{n}=I \oplus J$, PF is bounded, QF satisfies (1.1), where $P: \mathbb{K}^{n} \rightarrow I, Q: \mathbb{K}^{n} \rightarrow J$ are the natural projections. 
If we replace $\|\cdot\|$ by the usual norm $\|\cdot\|_{u}$ on $\mathbb{K}^{n}$ defined by

$$
\left\|\left(a_{1}, a_{2}, \ldots, a_{n}\right)\right\|_{u}=\sqrt{\left|a_{1}\right|^{2}+\left|a_{2}\right|^{2}+\cdots+\left|a_{n}\right|^{2}},
$$

we can estimate $P F$ (in Theorem 1.2 and Theorem 1.3) as follows.

Theorem 1.4 The following two statements are valid.

(a) If $F: G \rightarrow \mathbb{K}^{n}, F=\left(f_{1}, f_{2}, \ldots, f_{n}\right)$ satisfies (1.4), then PF satisfies

$$
\|P F(y)\|_{u} \leq \frac{\sqrt{|L|}}{2}(1+\sqrt{1+4 \phi(y)})
$$

for all $y \in G$, where $|L|$ denotes the number of the elements of $L$. In particular, if $|L|=1$ and $G$ is a group, then PF satisfies either

$$
\frac{1}{2}(1+\sqrt{1-4 \phi(y)}) \leq\|P F(y)\|_{u} \leq \frac{1}{2}(1+\sqrt{1+4 \phi(y)})
$$

for all $y \in B:=\left\{y \in G: \phi(y)<\frac{1}{4}\right\}$, or

$$
\|P F(y)\|_{u} \leq \frac{1}{2}(1-\sqrt{1-4 \phi(y)})
$$

for all $y \in B$.

(b) If $F: G \rightarrow \mathbb{K}^{n}, F=\left(f_{1}, f_{2}, \ldots, f_{n}\right)$ satisfies (1.5), then PF satisfies (1.7). In particular if $G$ is a group, $g$ is surjective and $|L|=1$, then PF satisfies (1.8) or (1.9).

\section{Proofs}

Let $g: G \rightarrow G$ and $\phi: G \rightarrow[0, \infty)$ be given. We first consider the stability of the functional equation

$$
f(x \circ g(y))-f(x) f(y)=0
$$

in the class of functions $f: G \rightarrow \mathbb{K}$, i.e., we investigate both bounded and unbounded functions $f: G \rightarrow \mathbb{K}$ satisfying the functional inequalities

$$
\begin{aligned}
& |f(x \circ g(y))-f(x) f(y)| \leq \phi(y), \\
& |f(x \circ g(y))-f(x) f(y)| \leq \phi(x)
\end{aligned}
$$

for all $x, y \in G$.

Lemma 2.1 Assume that $g=\sigma$ is an involution and $f: G \rightarrow \mathbb{K}$ is an unbounded function satisfying the inequality (2.2). Then $f$ is exponential and satisfies (2.1). In particular, if $G$ is 2-divisible, then $f$ has the form

$$
f(x)=m\left(\frac{x \circ \sigma(x)}{2}\right)
$$

for all $x \in G$, where $m: G \rightarrow \mathbb{K}$ is an exponential function. 
Proof Choose a sequence $x_{n} \in G, n=1,2,3, \ldots$, such that $\left|f\left(x_{n}\right)\right| \rightarrow \infty$ as $n \rightarrow \infty$. Putting $x=x_{n}, n=1,2,3, \ldots$, in (2.2), dividing the result by $\left|f\left(x_{n}\right)\right|$ and letting $n \rightarrow \infty$ we have

$$
f(y)=\lim _{n \rightarrow \infty} \frac{f\left(x_{n} \circ \sigma(y)\right)}{f\left(x_{n}\right)}
$$

for all $y \in G$. Multiplying both sides of (2.5) by $f(x)$ and using (2.2) and (2.5) we have

$$
\begin{aligned}
f(y) f(x) & =\lim _{n \rightarrow \infty} \frac{f\left(x_{n} \circ \sigma(y)\right) f(x)}{f\left(x_{n}\right)}=\lim _{n \rightarrow \infty} \frac{f\left(x_{n} \circ \sigma(y) \circ \sigma(x)\right)}{f\left(x_{n}\right)} \\
& =\lim _{n \rightarrow \infty} \frac{f\left(x_{n} \circ \sigma(y \circ x)\right)}{f\left(x_{n}\right)}=f(y \circ x)
\end{aligned}
$$

for all $x, y \in G$. Thus, $f$ is an exponential function, say $f=m$. From (2.2) and (2.6) we have

$$
|f(x)||f(\sigma(y))-f(y)| \leq \phi(y)
$$

for all $x, y \in G$. Since $f$ is unbounded, from (2.7) we have

$$
f(\sigma(y))=f(y)
$$

for all $y \in G$. Replacing $y$ by $\sigma(y)$ in (2.6) and using (2.8) we get the equation (2.1). In particular, if $G$ is 2-divisible, then we can write

$$
\begin{aligned}
f(x) & =f\left(\frac{x}{2} \circ \frac{x}{2}\right)=f\left(\frac{x}{2} \circ \sigma\left(\frac{x}{2}\right)\right) \\
& =f\left(\frac{x}{2} \circ \frac{\sigma(x)}{2}\right)=m\left(\frac{x \circ \sigma(x)}{2}\right)
\end{aligned}
$$

for all $x \in G$. This completes the proof.

Lemma 2.2 Let $f: G \rightarrow \mathbb{K}$ be an unbounded function satisfying (2.2). Assume that there exists a sequence $x_{n}, n=1,2,3, \ldots$, satisfying

$$
\lim _{n \rightarrow \infty} \frac{\left|f\left(x_{n}\right)\right|}{1+\phi\left(x_{n}\right)}=\infty
$$

Then $f$ satisfies (2.1).

Proof Note that (2.10) implies

$$
\lim _{n \rightarrow \infty} \frac{1}{\left|f\left(x_{n}\right)\right|}=0 \quad \text { and } \quad \lim _{n \rightarrow \infty} \frac{\phi\left(x_{n}\right)}{\left|f\left(x_{n}\right)\right|}=0
$$

Putting $y=x_{n}, n=1,2,3, \ldots$, in $(2.2)$ and dividing the result by $\left|f\left(x_{n}\right)\right|$ we have

$$
\left|f(x)-\frac{f\left(x \circ g\left(x_{n}\right)\right)}{f\left(x_{n}\right)}\right| \leq \frac{\phi\left(x_{n}\right)}{\left|f\left(x_{n}\right)\right|}
$$


for all $x \in G, n=1,2,3, \ldots$. Letting $n \rightarrow \infty$ in (2.11) we have

$$
f(x)=\lim _{n \rightarrow \infty} \frac{f\left(x \circ g\left(x_{n}\right)\right)}{f\left(x_{n}\right)}
$$

for all $x \in G$. Multiplying both sides of (2.12) by $f(y)$ and using (2.2) and (2.12) we have

$$
\begin{aligned}
f(x) f(y) & =\lim _{n \rightarrow \infty} \frac{f\left(x \circ g\left(x_{n}\right)\right) f(y)}{f\left(x_{n}\right)}=\lim _{n \rightarrow \infty} \frac{f\left(x \circ g\left(x_{n}\right) \circ g(y)\right)}{f\left(x_{n}\right)} \\
& =\lim _{n \rightarrow \infty} \frac{f\left(x \circ g(y) \circ g\left(x_{n}\right)\right)}{f\left(x_{n}\right)}=f(x \circ g(y))
\end{aligned}
$$

for all $x, y \in G$. This completes the proof.

Lemma 2.3 Assume that $g$ is bijective and $f: G \rightarrow \mathbb{K}$ is an unbounded function satisfying the inequality (2.2). Then $f \circ g^{-1}$ is an exponential function.

Proof Choose a sequence $x_{n} \in G, n=1,2,3, \ldots$, such that $\left|f\left(x_{n}\right)\right| \rightarrow \infty$ as $n \rightarrow \infty$. Putting $x=x_{n}, n=1,2,3, \ldots$, in $(2.2)$, dividing the result by $\left|f\left(x_{n}\right)\right|$, replacing $y$ by $g^{-1}(y)$ and letting $n \rightarrow \infty$ we have

$$
f\left(g^{-1}(y)\right)=\lim _{n \rightarrow \infty} \frac{f\left(x_{n} \circ y\right)}{f\left(x_{n}\right)}
$$

for all $y \in G$. Multiplying both sides of (2.14) by $f\left(g^{-1}(x)\right)$ and using (2.2) and (2.14) we have

$$
\begin{aligned}
f\left(g^{-1}(y)\right) f\left(g^{-1}(x)\right) & =\lim _{n \rightarrow \infty} \frac{f\left(x_{n} \circ y\right) f\left(g^{-1}(x)\right)}{f\left(x_{n}\right)} \\
& =\lim _{n \rightarrow \infty} \frac{f\left(x_{n} \circ y \circ x\right)}{f\left(x_{n}\right)}=f\left(g^{-1}(y \circ x)\right)
\end{aligned}
$$

for all $x, y \in G$. Thus, $f \circ g^{-1}$ is an exponential function. This completes the proof.

Proof of Theorem 1.2 Since every two norms in $K^{n}$ are equivalent, from (1.4) there exists $\alpha>0$ such that

$$
\begin{aligned}
\left|f_{j}(x \circ g(y))-f_{j}(x) f_{j}(y)\right| & \leq\|F(x \circ g(y))-F(x) F(y)\|_{u} \\
& \leq \alpha\|F(x \circ g(y))-F(x) F(y)\| \leq \alpha \phi(y)
\end{aligned}
$$

for all $x, y \in G$ and all $j \in\{1,2, \ldots, n\}$. For the case (i), by Lemma 2.1, $f_{j}$ satisfies (2.1) for all $j \in K$. For the case (ii), by Lemma $2.2, f_{j}$ satisfies (2.1) for all $j \in K$. Let $I=\left\{\left(a_{1}, a_{2}, \ldots, a_{n}\right)\right.$ : $a_{i}=0$ for $\left.i \in K\right\}, J=\left\{\left(a_{1}, a_{2}, \ldots, a_{n}\right): a_{i}=0\right.$ for $\left.i \in L\right\}$. Then it follows that $\mathbb{K}^{n}=I \oplus J, P F$ is bounded and $Q F$ satisfies (1.1). If $g$ is bijective, then by Lemma 2.3, $f_{j} \circ g^{-1}$ are exponential function for all $j \in K$, which implies $Q\left(F \circ g^{-1}\right)$ is an exponential function. This completes the proof.

Lemma 2.4 Assume that $g=\sigma$ is an involution and $f: G \rightarrow \mathbb{K}$ is an unbounded function satisfying the inequality (2.3). Then $f$ satisfies (2.1). In particular, if $G$ is 2-divisible, then $f$ 
has the form

$$
f(x)=m\left(\frac{x \circ \sigma(x)}{2}\right)
$$

for all $x \in G$, where $m: G \rightarrow \mathbb{K}$ is an exponential function.

Proof Choose a sequence $y_{n} \in G, n=1,2,3, \ldots$, such that $\left|f\left(y_{n}\right)\right| \rightarrow \infty$ as $n \rightarrow \infty$. Putting $y=y_{n}, n=1,2,3, \ldots$, in (2.3), dividing the result by $\left|f\left(y_{n}\right)\right|$ and letting $n \rightarrow \infty$ we have

$$
f(x)=\lim _{n \rightarrow \infty} \frac{f\left(x \circ \sigma\left(y_{n}\right)\right)}{f\left(y_{n}\right)} .
$$

Putting $x=e$ in (2.3) and replacing $y$ by $\sigma(y)$ in the result we have

$$
|f(y)-f(e) f(\sigma(y))| \leq \phi(e)
$$

for all $x, y \in G$. Multiplying both sides of (2.18) by $f(y)$ and using (2.3), (2.18), and (2.19) we have

$$
\begin{aligned}
f(y) f(x) & =\lim _{n \rightarrow \infty} \frac{f(y) f\left(x \circ \sigma\left(y_{n}\right)\right)}{f\left(y_{n}\right)}=\lim _{n \rightarrow \infty} \frac{f\left(y \circ \sigma\left(x \circ \sigma\left(y_{n}\right)\right)\right)}{f\left(y_{n}\right)} \\
& =\lim _{n \rightarrow \infty} \frac{f(e) f\left(\sigma(y) \circ x \circ \sigma\left(y_{n}\right)\right)}{f\left(y_{n}\right)}=f(e) f(\sigma(y) \circ x)
\end{aligned}
$$

for all $x, y \in G$. Putting $x=e$ in (2.20) we have

$$
f(y)=f(\sigma(y))
$$

for all $y \in$ G. From (2.19) and (2.21) we have

$$
|f(y)||1-f(e)| \leq \phi(e)
$$

for all $y \in G$. Since $f$ is unbounded, from (2.22) we have $f(e)=1$. Thus, $f$ satisfies (2.1). This completes the proof.

Proof of Theorem 1.3 From (1.5), as in (2.16) there exists $\alpha>0$ such that

$$
\left|f_{j}(x \circ g(y))-f_{j}(x) f_{j}(y)\right| \leq \alpha \phi(x)
$$

for all $x, y \in G, j \in\{1,2, \ldots, n\}$. Applying Lemma 2.4 to (2.23) for each $j \in K$ we find that $f_{j}$ satisfies (2.1) for all $j \in K$, which implies that $Q F$ satisfies (1.1). This completes the proof.

Now, we investigate bounded functions satisfying each of (2.2) and (2.3) (see [4, 11-13] for bounded solutions of an exponential functional equation). 
Lemma 2.5 Let $f: G \rightarrow \mathbb{K}$ be a bounded function satisfying (2.2). Then $f$ satisfies

$$
|f(y)| \leq \frac{1}{2}(1+\sqrt{1+4 \phi(y)})
$$

for all $y \in G$. In particular, $G$ is a group and let $B=\left\{y \in G: \phi(y)<\frac{1}{4}\right\}$, then $f$ satisfies either

$$
\frac{1}{2}(1+\sqrt{1-4 \phi(y)}) \leq|f(y)| \leq \frac{1}{2}(1+\sqrt{1+4 \phi(y)})
$$

for all $y \in B$, or

$$
|f(y)| \leq \frac{1}{2}(1-\sqrt{1-4 \phi(y)})
$$

for all $y \in B$.

Proof Let $M_{f}=\sup _{x \in G}|f(x)|$. Using the triangle inequality with (2.2) we have

$$
|f(x) f(y)| \leq|f(x \circ g(y))|+\phi(y) \leq M_{f}+\phi(y)
$$

for all $x, y \in G$. Taking the supremum of the left hand side of (2.27) with respect to $x \in G$ we get $M_{f}|f(y)| \leq M_{f}+\phi(y)$ for all $y \in G$. Thus, we have

$$
M_{f}(|f(y)|-1) \leq \phi(y)
$$

for all $y \in G$. From (2.28) we have

$$
|f(y)|(|f(y)|-1) \leq \phi(y)
$$

for all $y \in G$. Solving the inequality (2.29) we get (2.24). Now, we assume that $G$ is a group. Replacing $x$ by $x \circ g(y)^{-1}$ in (2.2) and using the triangle inequality we have

$$
|f(x)| \leq\left|f\left(x \circ g(y)^{-1}\right) f(y)\right|+\phi(y) \leq M_{f}|f(y)|+\phi(y)
$$

for all $x, y \in G$. Taking the supremum of the left hand side of (2.30) with respect to $x \in G$ we get $M_{f} \leq M_{f}|f(y)|+\phi(y)$ for all $y \in G$. Thus, we have

$$
M_{f}(1-|f(y)|) \leq \phi(y)
$$

for all $y \in G$. From (2.28) and (2.31) we have

$$
|f(y)||1-| f(y)|| \leq M_{f}|1-| f(y)|| \leq \phi(y)
$$

for all $y \in G$. For each fixed $y \in B$, solving the inequality (2.32) we get

$$
\frac{1}{2}(1+\sqrt{1-4 \phi(y)}) \leq|f(y)| \leq \frac{1}{2}(1+\sqrt{1+4 \phi(y)}),
$$


or

$$
|f(y)| \leq \frac{1}{2}(1-\sqrt{1-4 \phi(y)})
$$

Now, assume that there exist a bounded function $f$ and $y_{1}, y_{2} \in B$ such that

$$
\left|f\left(y_{1}\right)\right| \leq \frac{1}{2}\left(1-\sqrt{1-4 \phi\left(y_{1}\right)}\right), \quad\left|f\left(y_{2}\right)\right| \geq \frac{1}{2}\left(1+\sqrt{1-4 \phi\left(y_{2}\right)}\right) .
$$

Then from (2.31) we have

$$
\left|f\left(y_{2}\right)\right|\left(1-\left|f\left(y_{1}\right)\right|\right) \leq M_{f}\left(1-\left|f\left(y_{1}\right)\right|\right) \leq \phi\left(y_{1}\right)
$$

On the other hand, from (2.35) we have

$$
\begin{aligned}
\left|f\left(y_{2}\right)\right|\left(1-\left|f\left(y_{1}\right)\right|\right) \geq & \frac{1}{2}\left(1+\sqrt{1-4 \phi\left(y_{2}\right)}\right)\left(1-\frac{1}{2}\left(1-\sqrt{1-4 \phi\left(y_{1}\right)}\right)\right) \\
& >\frac{1}{2}\left(1-\sqrt{1-4 \phi\left(y_{1}\right)}\right)\left(1-\frac{1}{2}\left(1-\sqrt{1-4 \phi\left(y_{1}\right)}\right)\right)=\phi\left(y_{1}\right),
\end{aligned}
$$

which contradicts (2.36). Thus, $f$ satisfies (2.25) for all $y \in B$, or it satisfies (2.26) for all $y \in B$. This completes the proof.

Lemma 2.6 Let $f: G \rightarrow \mathbb{K}$ be a bounded function satisfying (2.3). Then $f$ satisfies (2.24) for all $y \in G$. In particular, if $G$ is a group and $g$ is surjective, then $f$ satisfies (2.25) for all $y \in B:=\left\{y \in G: \phi(y)<\frac{1}{4}\right\}$, or satisfies (2.26) for all $y \in B$.

Proof Using the triangle inequality with (2.3) we have

$$
|f(x) f(y)| \leq|f(x \circ g(y))|+\phi(x) \leq M_{f}+\phi(x)
$$

for all $x, y \in G$. Taking the supremum of the left hand side of (2.37) with respect to $y \in G$ we get $M_{f}|f(x)| \leq M_{f}+\phi(x)$ for all $x \in G$. Thus, we have

$$
M_{f}(|f(x)|-1) \leq \phi(x)
$$

for all $x \in G$. From (2.38) we get (2.24) as in the proof of Lemma 2.5. We assume that $G$ is a group. For given $x, z \in G$, choosing $w \in G$ such that $g(w)=x^{-1} \circ z$, putting $y=w$ in (2.3) and using the triangle inequality we have

$$
|f(z)| \leq|f(x) f(w)|+\phi(x) \leq|f(x)| M_{f}+\phi(x)
$$

for all $x, z \in G$. Taking the supremum of the left hand side of (2.39) we get $M_{f} \leq M_{f}|f(x)|+$ $\phi(x)$ for all $x \in G$. Thus, we have

$$
M_{f}(1-|f(x)|) \leq \phi(x)
$$

for all $x \in G$. Now, the remaining parts of the proof are the same as those of Lemma 2.5. 
Proof of Theorem 1.4 From Lemma 2.5 and Lemma 2.6, for each $j \in L$ we have

$$
\left|f_{j}(y)\right| \leq \frac{1}{2}(1+\sqrt{1+4 \phi(y)})
$$

for all $y \in G$. Thus, from (2.41) we have

$$
\|P F(y)\|_{u}=\sqrt{\sum_{j \in L}\left|f_{j}(y)\right|^{2}} \leq \frac{\sqrt{|L|}}{2}(1+\sqrt{1+4 \phi(y)})
$$

for all $y \in G$, which gives (1.7). Now, if $|L|=1$, say $L=\left\{j_{0}\right\}$ we have

$$
\|P F(y)\|_{u}=\left|f_{j_{0}}(y)\right|
$$

for all $y \in G$. Thus, the inequalities (1.8) and (1.9) follow immediately from (2.25) and (2.26). This completes the proof.

\section{Competing interests}

The author declares that he has no competing interests.

\section{Author's contributions}

The author is the only person who is responsible to this work.

\section{Acknowledgements}

The author is very thankful to the referees for valuable suggestions that improved the presentation of the paper. This work was supported by Basic Science Research Program through the National Foundation of Korea (NRF) funded by the Korea Government (no. 2012R1A1A008507).

Received: 6 March 2014 Accepted: 23 May 2014 Published: 30 May 2014

\section{References}

1. Ger, R, Šemrl, P: The stability of exponential equation. Proc. Am. Math. Soc. 124, 779-787 (1996)

2. Najdecki, A: On stability of functional equation connected with the Reynolds operator. J. Inequal. Appl. 2007, Article ID 79816 (2007)

3. Aczél, J, Dhombres, J: Functional Equations in Several Variables. Cambridge University Press, New York (1989)

4. Baker, JA: The stability of cosine functional equation. Proc. Am. Math. Soc. 80, 411-416 (1980)

5. Brzdęk, J: On solutions of a generalization of the Reynolds functional equation. Demonstr. Math. 41, 859-868 (2008)

6. Brzdekk, J, Najdecki, A, Xu, B: Two general theorems on superstability of functional equations. Aequ. Math. doi:10.1007/s0010-014-0266-6

7. Chudziak, J, Tabor, J: On the stability of the Gołąb-Schinzel functional equation. J. Math. Anal. Appl. 302, 196-200 (2005)

8. Marras, Y: Sur l'équation fonctionnelle $f[x f(y)]=f(x) \cdot f(y)$. Bull. Cl. Sci., Acad. R. Belg. 5e Série 55 779-787 (1969)

9. Brillouët-Belluot, N, Brzdek, J, Ciepliński, K: On some recent developments in Ulam's type stability. Abstr. Appl. Anal. 2012, Article ID 716936 (2012)

10. Brzdek, J, Ciepliński, K: Hyperstability and superstability. Abstr. Appl. Anal. 2013, Article ID 401756 (2013)

11. Albert, M, Baker, JA: Bounded solutions of a functional inequality. Can. Math. Bull. 25, 491-495 (1982)

12. Chung, J: On an exponential functional inequality and its distributional version. Can. Math. Bull. (2012). doi:10.4153/CMB-2014-012-x

13. Hyers, DH, Isac, G, Rassias, TM: Stability of Functional Equations in Several Variables. Birkhäuser, Basel (1998)

10.1186/1687-1847-2014-158

Cite this article as: Chung: Stability of a functional equation connected with Reynolds operator. Advances in Difference Equations 2014, 2014:158 